\title{
$B$ DECAYS TO OPEN AND HIDDEN CHARM AT BELLE
}

\author{
A. DRUTSKOY \\ Physics Department, University of Cincinnati, \\ 345 College Court, Cincinnati, OH 45221, USA
}

\begin{abstract}
The recent Belle collaboration measurements of $B$ decays to open and hidden charm are discussed. Color-suppressed decay branching fractions are measured with an improved accuracy. The branching fractions of the $\bar{B}^{0} \rightarrow D_{s}^{+} K^{-}$and $\bar{B}^{0} \rightarrow D_{s}^{-} \pi^{+}$decays, measured with improved accuracy, and $\bar{B}^{0} \rightarrow D_{s J}^{+} K^{-}$and $\bar{B}^{0} \rightarrow D_{s J}^{-} \pi^{+}$decays, measured for the first time, are compared. The two-body invariant masses of the three-body $B^{0} \rightarrow D^{(*) 0} \pi^{+} \pi^{-}$and $B^{0} \rightarrow J / \Psi \pi^{+} \pi^{-}$decays are studied.
\end{abstract}

\section{Results}

\subsection{Color-suppressed $\bar{B}^{0} \rightarrow D^{0} \pi^{0}(/ \omega / \eta)$ and $\bar{B}^{0} \rightarrow D^{(*) 0} \eta^{\prime}$ decays}

The weak decays $\bar{B}^{0} \rightarrow D^{(*) 0} h^{0}$, where $h^{0}$ represents a light neutral meson, are usually described by a "color-suppressed" diagram as shown in Fig.1.

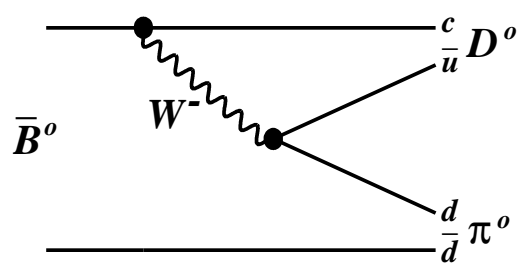

Figure 1. Color-suppressed diagram for the decay $\bar{B}^{0} \rightarrow D^{(*) 0} \pi^{0}$.

Within "näive" factorization models ${ }^{1,2}$, the color-matching requirement leads to branching fractions in the range $(0.3-1.7) \times 10^{-4}$. However previous measurements by the CLEO ${ }^{3}$, Belle ${ }^{4}$ and BaBar ${ }^{5}$ collaborations were substantially shifted to the larger values $(2-4) \times 10^{-4}$. The new Belle measurements are based on a larger data sample of $140 \mathrm{fb}^{-1}$. This corresponds to a seven-fold increase over the previous Belle measurement and almost twice that of the earlier BaBar measurement. With increased statistics a more detailed study of continuum and $B \bar{B}$ related backgrounds was done, re- ducing systematic uncertainties.

The Belle measurements of these branching fractions for each decay mode is shown in Table 1.

Table 1. $B$ branching fractions for color-suppressed decays.

\begin{tabular}{|l|c|}
\hline Decay mode & Br. Fraction $\left(\times 10^{-4}\right)$ \\
\hline $\bar{B}^{0} \rightarrow D^{0} \pi^{0}$ & $2.31 \pm 0.12 \pm 0.23$ \\
$\bar{B}^{0} \rightarrow D^{0} \eta(\gamma \gamma)$ & $1.77 \pm 0.18 \pm 0.20$ \\
$\bar{B}^{0} \rightarrow D^{0} \eta\left(\pi^{0} \pi \pi\right)$ & $1.89 \pm 0.29 \pm 0.20$ \\
$\bar{B}^{0} \rightarrow D^{0} \eta$ & $1.83 \pm 0.15 \pm 0.27$ \\
$\bar{B}^{0} \rightarrow D^{0} \omega\left(\pi^{0} \pi \pi\right)$ & $2.25 \pm 0.21 \pm 0.28$ \\
$\bar{B}^{0} \rightarrow D^{0} \eta^{\prime}$ & $1.17 \pm 0.20{ }_{-0.14}^{+0.10}$ \\
$\bar{B}^{0} \rightarrow D^{* 0} \eta^{\prime}$ & $1.23 \pm 0.34 \pm 0.21$ \\
\hline
\end{tabular}

These branching fractions are about one-two standard deviations lower than the BaBar measurements but higher than early predictions within factorization models. The latter discrepancy may be explained by additional contributions from final state rescattering or non-factorisable diagrams.

\subsection{Improved measurement of $\bar{B}^{0} \rightarrow D_{s}^{+} K^{-}$and $\bar{B}^{0} \rightarrow D_{s}^{-} \pi^{+}$ decays and first study of $\bar{B}^{0} \rightarrow D_{s J}^{+} K^{-}$and $\bar{B}^{0} \rightarrow D_{s J}^{-} \pi^{+}$ decays}

The decays $\bar{B}^{0} \rightarrow D_{s(J)}^{+} K^{-}$are of special interest because the quark content of the initial $\bar{B}^{0}$ meson $(b \bar{d})$ is completely differ- 

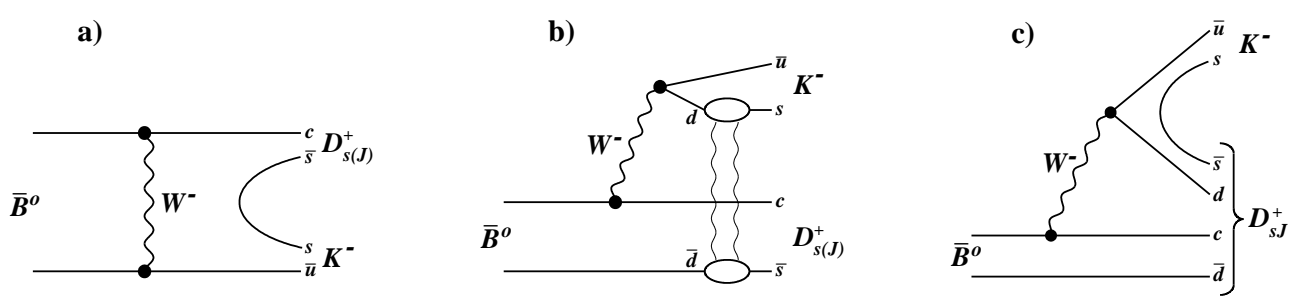

Figure 2. Diagrams describing $\bar{B}^{0} \rightarrow D_{s J}^{+} K^{-}$decay.

ent from that of the $D_{s(J)}^{+} K^{-}$final state $(\operatorname{cs} \bar{s} \bar{u})$, indicating an unusual configuration with both initial quarks involved in the weak decay. Branching fractions with the $D_{s}^{+}$meson $\mathcal{B}\left(\bar{B}^{0} \rightarrow D_{s}^{+} K^{-}\right)=\left(4.6_{-1.1}^{+1.2} \pm 1.3\right) \cdot 10^{-5}$ and $(3.2 \pm 1.0 \pm 1.0) \cdot 10^{-5}$ have been measured by the Belle ${ }^{6}$ and BaBar ${ }^{7}$ collaborations, respectively. Predictions of this branching fraction have been obtained assuming a dominant contribution from a PQCD factorization $W$ exchange process ${ }^{8,9}$ (Fig. 2a) or, alternatively, from final state interactions ${ }^{10,11}$ (Fig. 2b), and range from a few units of $10^{-6}$ to $10^{-4}$. If the $D_{s . J}$ mesons have a fourquark component then the tree diagram with $s \bar{s}$ pair creation (shown in Fig. 2c) may also contribute.

The decay mode $\bar{B}^{0} \rightarrow D_{s(J)}^{-} \pi^{+}$can be described by a " $b$ to $u$ " tree diagram. Within the factorization approach ${ }^{12}$ the branching fraction ratio $R_{\pi^{+} / D^{+}}=\mathcal{B}\left(\bar{B}^{0} \rightarrow\right.$ $\left.D_{s}^{-} \pi^{+}\right) / \mathcal{B}\left(\bar{B}^{0} \rightarrow D_{s}^{-} D^{+}\right)$is predicted to be $(0.424 \pm 0.041) \cdot\left|V_{u b} / V_{c b}\right|^{2}$ and can be used to obtain the ratio of Cabbibo-KobayashiMaskawa matrix elements $\left|V_{u b} / V_{c b}\right|$.

The $B$ decay channels with the pseudoscalar $D_{s}$ meson were studied using 253 $\mathrm{fb}^{-1}$ of data $\left(275 \times 10^{6} B \bar{B}\right.$ pairs $)$, whereas channels with the $D_{s J}$ were studied using $140 \mathrm{fb}^{-1}$ of data $\left(152 \times 10^{6} B \bar{B}\right.$ pairs $) . D_{s}^{+}$ mesons are reconstructed in the $\phi \pi^{+}, K^{* 0} K^{+}$ and $K_{S}^{0} K^{+}$decay channels. $D_{s J}$ mesons are reconstructed in the $D_{s J}^{*}(2317)^{+} \rightarrow D_{s}^{+} \pi^{0}$ and $D_{s J}(2460)^{+} \rightarrow D_{s}^{+} \gamma$ decay modes.
The $D_{s}$ mass distributions in the $B$ signal region are shown in Fig. 3. The points are experimental data and the curves display the fit results. In addition to clear signals at the $D_{s}^{+}$mass in Fig. 3, the $D^{+}$mass peak $\left(D^{+}\right.$ decays in the same final states) is also seen.

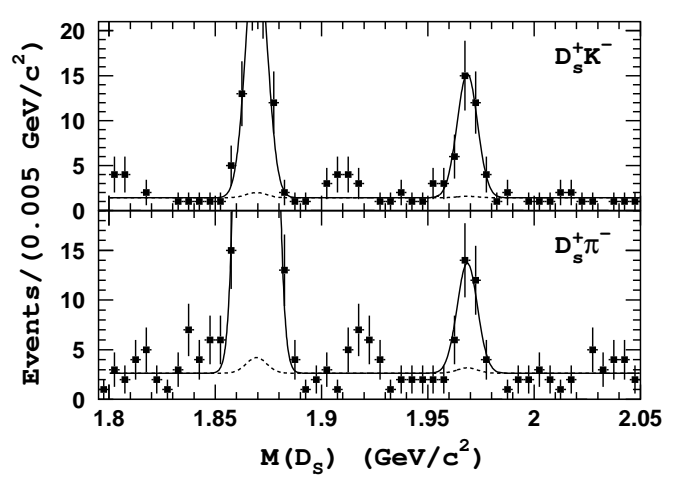

Figure 3. $M\left(D_{s}\right)$ spectra for $\bar{B}^{0} \rightarrow D_{s}^{+} K^{-}$(top) and $\bar{B}^{0} \rightarrow D_{s}^{-} \pi^{+}$(bottom) in the $B$ signal region.

The branching fractions obtained from the fits are $\mathcal{B}\left(\bar{B}^{0} \rightarrow D_{s}^{+} K^{-}\right)=(2.93 \pm$ $0.55 \pm 0.79) \cdot 10^{-5}$ and $\mathcal{B}\left(\bar{B}^{0} \rightarrow D_{s}^{-} \pi^{+}\right)=$ $(1.94 \pm 0.47 \pm 0.52) \cdot 10^{-5}$. These agree within errors with the previous measurements.

The $\Delta M\left(D_{s J}\right)$ distributions in the $B$ signal region for various $D_{s J}^{+} K^{-}$and $D_{s J}^{-} \pi^{+}$ combinations are shown in Fig. 4. A clear $\bar{B}^{0} \rightarrow D_{s J}^{*}(2317)^{+} K^{-}$signal is observed; no significant signals are observed in the remaining modes.

For the $\bar{B}^{0} \rightarrow D_{s J}^{*}(2317)^{+} K^{-}$decay, the product branching fraction is measured to be 


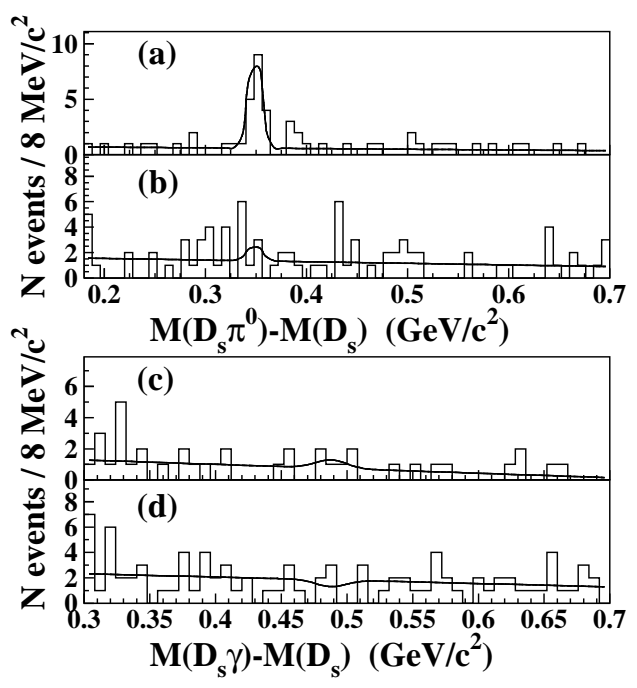

Figure 4. The $\Delta M\left(D_{s J}\right)$ distributions in the $B$ signal region for the (a) $D_{s J}^{*}(2317)^{+} K^{-}$, (b) $D_{s J}^{*}(2317)^{-} \pi^{+}$, (c) $D_{s J}(2460)^{+} K^{-}$and (d) $D_{s J}(2460)^{-} \pi^{+}$combinations are shown.

$\mathcal{B}\left(\bar{B}^{0} \rightarrow D_{s J}^{*}(2317)^{+} K^{-}\right) \times \mathcal{B}\left(D_{s J}^{*}(2317)^{+} \rightarrow\right.$ $\left.D_{s}^{+} \pi^{0}\right)=\left(5.3_{-1.3}^{+1.5} \pm 0.7 \pm 1.4\right) \cdot 10^{-5}$. Recent measurements imply that the $D_{s J}^{*}(2317)^{+} \rightarrow$ $D_{s}^{+} \pi^{0}$ channel is dominant and the $D_{s J}(2460)^{+} \rightarrow D_{s}^{+} \gamma$ fraction is around $30 \%$. Taking into account these approximate values, we can conclude that $\mathcal{B}\left(\bar{B}^{0} \rightarrow\right.$ $\left.D_{s J}^{*}(2317)^{+} K^{-}\right)$is of the same order of magnitude as $\mathcal{B}\left(\bar{B}^{0} \rightarrow D_{s}^{+} K^{-}\right)$, but at least a factor two larger than the $\bar{B}^{0} \rightarrow D_{s J}(2460)^{+} K^{-}$ branching fraction, in contrast to the naïve expectation that decays with the same spindoublet $D_{s J}^{*}(2317)^{+}$and $D_{s J}(2460)^{+}$mesons would have similar rates. It is interesting to mention, that the ratio of $\mathcal{B}(B \rightarrow$ $\left.D_{s J}^{*}(2317)^{+} D\right)$ and $\mathcal{B}\left(B \rightarrow D_{s}^{+} D\right)$ decay branching fractions ${ }^{13}$ is approximately $1 / 10$, indicating to a different behaviours of the $B \rightarrow D_{s} K$ and $B \rightarrow D_{s} D$ processes ${ }^{14}$.

\subsection{Study of $B^{0} \rightarrow D^{(*) 0} \pi^{+} \pi^{-}$decays}

The three-body $B^{0} \rightarrow D^{(*) 0} \pi^{+} \pi^{-}$decays were studied with $140 \mathrm{fb}^{-1}$ of data. These processes can provide important in- formation about intermediate two-body resonances. The $D^{0} \pi^{+}$and $D^{* 0} \pi^{+}$mass distributions are shown in Figs. 5,6. Points are experimental data, the hatched histogram is obtained using sideband events and the open histogram is MC simulation with all intermediate resonances included. The masses and widths of narrow $\left(\leq 100 \mathrm{MeV} / \mathrm{c}^{2}\right) D^{* *}$ resonances observed are listed in Table 2 . These values are in a good agreement with predictions obtained within potential models ${ }^{15}$.

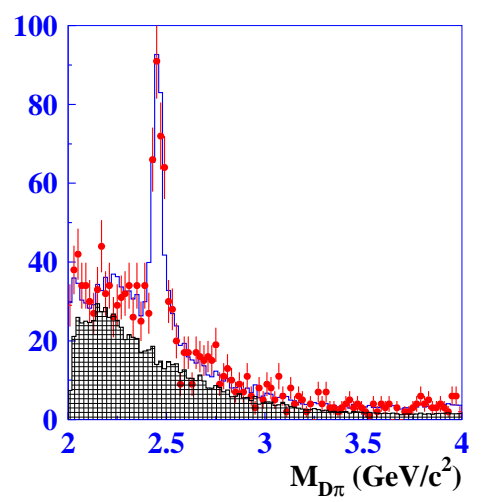

Figure 5. The $D^{0} \pi^{+}$mass distribution.

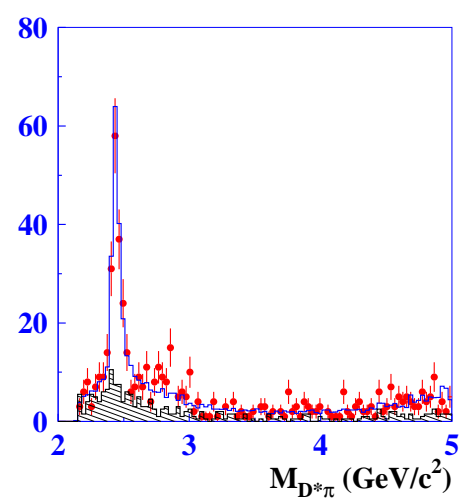

Figure 6 . The $D^{* 0} \pi^{+}$mass distribution.

The quasi-two-body $\bar{B}^{0} \rightarrow D^{* *+} \pi^{-}$final state branching fractions were measured and compared to results obtained by the Belle collaboration in the studies of the charged $B^{+} \rightarrow D^{(*)-} \pi^{+} \pi^{+}$decay modes ${ }^{16}$. The branching fractions obtained for the narrow resonances are similar for the neutral and 
Table 2. Masses and widths of narrow $D^{* *}$ resonances.

\begin{tabular}{|l|c|}
\hline Parameter & Value, $\mathrm{MeV} / \mathrm{c}^{2}$ \\
\hline$M\left(D_{2}^{*+}\right)$ & $2459.5 \pm 2.3 \pm 0.7_{-0.5}^{+4.9}$ \\
\hline$\Gamma\left(D_{2}^{*+}\right)$ & $48.9 \pm 5.4 \pm 4.2 \pm 1.9$ \\
\hline$M\left(D_{1}^{+}\right)$ & $2428.2 \pm 2.9 \pm 1.6 \pm 0.6$ \\
\hline$\Gamma\left(D_{1}^{+}\right)$ & $34.9 \pm 6.6_{-0.9}^{+4.1} \pm 4.1$ \\
\hline
\end{tabular}

charged $B$ decays.

\subsection{Study of $B^{0} \rightarrow J / \Psi \pi^{+} \pi^{-}$decays}

The decay $B^{0} \rightarrow J / \Psi \rho^{0}$ is governed by the $b \rightarrow c \bar{c} d$ transition and can exhibit a $C P$ violating asymmetry. In contrast to the $b \rightarrow$ $c \bar{c} s$ transition, the $b \rightarrow c \bar{c} d$ process has substantial contributions from both the tree and penguin amplitudes, which could lead to different $C P$ asymmetries of these processes. Thus, $B^{0} \rightarrow J / \Psi \rho^{0}$ decays play an important role in probing non-tree diagram contributions.

The resonant structure of the $\pi^{+} \pi^{-}$invariant mass spectrum from $B^{0} \rightarrow J / \Psi \pi^{+} \pi^{-}$ decays was studied using $140 \mathrm{fb}^{-1}$ of data. Five types of events are considered in the fit shown in Fig. 7: (i) $B^{0} \rightarrow J / \Psi \rho^{0}$; (ii) $B^{0} \rightarrow$ $J / \Psi f_{2}$; (iii) $B^{0} \rightarrow J / \Psi \pi^{+} \pi^{-}$(non-resonant); (iv) $B^{0} \rightarrow J / \Psi K_{S}^{0}$ (background) and (v) combinatorial background. The branching fractions obtained are $\mathcal{B}\left(B^{0} \rightarrow J / \Psi \rho^{0}\right)=$ $(2.8 \pm 0.3 \pm 0.3) \times 10^{-5}$ and $\mathcal{B}\left(B^{0} \rightarrow J / \Psi f_{2}\right)=$ $(9.8 \pm 3.9 \pm 2.0) \times 10^{-6}$. The statistical significance of the latter is $2.9 \sigma$, and an upper limit is also set: $\mathcal{B}\left(B^{0} \rightarrow J / \Psi f_{2}\right)<$ $1.5 \times 10^{-5}$ at $90 \%$ C.L. An upper limit is also set on the non-resonant channel: $\mathcal{B}\left(B^{0} \rightarrow\right.$ $\left.J / \Psi\left(\pi^{+} \pi^{-}\right)_{\text {non-res. }}\right)<1.0 \times 10^{-5}$ at $90 \%$ C.L.

\section{References}

1. M. Neubert and A.A. Petrov, Phys. Lett. B 519, 50 (2001).

2. A. Deandrea and A.D. Polosa, Eur.

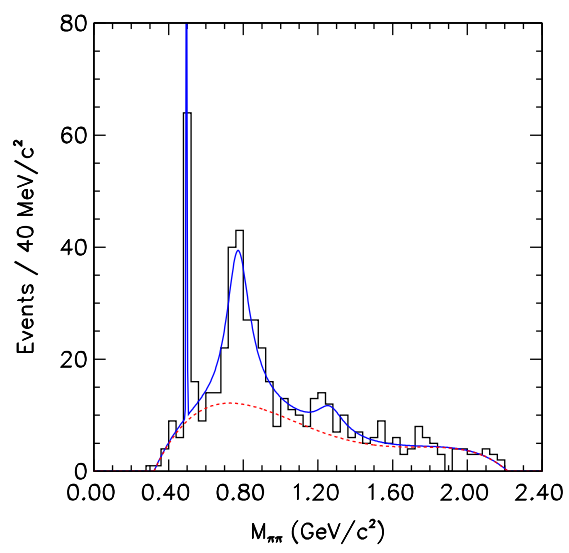

Figure 7. The distribution of $M_{\pi^{+} \pi^{-}}$for $B^{0} \rightarrow$ $J / \Psi \pi^{+} \pi^{-}$decay. Points are the experimental data. The solid line shows the result of fit with the $K_{S}^{0}$, $\rho^{0}, f_{2}$ and background contributions included, the dashed line is for the background contribution only.

Phys. Jour. C 22, 677 (2002).

3. CLEO Collaboration, T.E. Coan et al., Phys. Rev. Lett. 88, 062001 (2002).

4. Belle Collaboration, K. Abe et al., Phys. Rev. Lett. 88, 052002 (2002).

5. BaBar Collaboration, B. Aubert et al., Phys. Rev. D 69, 032004 (2004).

6. Belle Collaboration, P. Krokovny et al., Phys. Rev. Lett. 89, 231804 (2002).

7. BaBar Collaboration, B. Aubert et al., Phys. Rev. Lett. 90, 181803 (2003).

8. D. Du, L. Guo, D.-X. Zhang, Phys. Lett. B 406, 110 (1997).

9. C.D. Lu, hep-ph/0305061.

10. C.-K. Chua, W.-S. Hou, K.-C. Yang, Phys. Rev. D 65, 096007 (2002).

11. B. Blok, M. Gronau, J.L. Rosner, Phys. Rev. Lett. 78, 3999 (1997).

12. C.S. Kim, Y. Kwon, J. Lee, W. Namgung, Phys. Rev. D 63, 094506 (2001).

13. Belle Collaboration, P. Krokovny et al., Phys. Rev. Lett. 91, 262002 (2003).

14. C.-H. Chen, H.-n Li, Phys. Rev. D 69, 054002 (2004).

15. S. Godfrey and R. Kokoski, Phys. Rev. D 43, 1679 (1991).

16. Belle Collaboration, K. Abe et al., Phys. Rev. D 69, 112002 (2004). 